\title{
An Integrated Communication and Collaboration Platform for Distributed Scientific Workgroups
}

\author{
Christian Müller-Tomfelde ${ }^{1}$, Jane $\mathrm{Li}^{1,3}$, and Alex Hyatt ${ }^{2}$ \\ ${ }^{1}$ CSIRO ICT Centre, Australia \\ ${ }^{2}$ CSIRO Livestock Industries, Australia \\ ${ }^{3}$ University of Technology, Sydney, Australia \\ \{Christian.Mueller-Tomfelde, Jane.Li,Alex.Hyatt\}@csiro.au
}

\begin{abstract}
We present the design, technologies and user study of an advanced collaboration platform which integrates life-size video conferencing and group interactions on a large shared workspace. The platform has been developed to support the diagnostics and research scientists in an animal health laboratory to work collaboratively across a physical containment barrier. We present the design rationale for this enhanced shared workspace which allows the sharing of a range of data and synchronous interactions on computer applications in this complex work setting. This can not be simply supported by the "board-room" type of "telepresence" technology. We describe the technical solution which has focused on the ergonomic aspect and, importantly, the integration of communication and collaboration features in the shared workspace. The platform has been under routine use and a user study has shown that these design considerations are critical for supporting the distributed scientific collaborations and may be also applicable to other scientific domains.
\end{abstract}

Keywords: Human-Work Interaction Design, Interaction with Small or Large Displays, Computer-Supported Cooperative Work.

\section{Introduction}

Scientific work is collaborative in nature and collaboration technologies have been increasingly seen as important to enhance the scientific collaborations. Scientific collaborations have increasingly involved research teams which are distributed. To address the problem of geographic separation in research collaborations, "collaboratories" [1], "computer-supported system that allows scientists to work with each other, facilities, and database without regard to geographical location" [2], have been emerged over the past two decades in a diversity of scientific collaboration contexts, such as physical science, biological and health sciences [3]. These "e-Science", or "eResearch" applications came into focus with the fusion of computer and communication technologies and have demonstrated "the potential to dramatically enhance the output and productivity of researchers" [4] [5].

Designing technology to support the distributed scientific collaborations needs to move beyond developing general tools and audio-video communication 
mechanisms [5]. Scientific collaboration is driven by the need to share data and to exchange and increase knowledge about the data. The collaboration and communication are different to other work practice such as business meeting and lectures with regard to the type of material and amount of data scientists work with and the data sharing process. Various sources of data and information need to be "at hand" to be reviewed and interpreted by different experts at the same time rather than enabling one-to-many, lecture-style presentations.

Today's video conferencing technologies are highly developed in terms of audio and video quality and aiming for reproducing face-to-face situations, such as the specialized board meeting room for telepresence [6]. However these solutions may not be suitable to support scientific collaborations since they have limited support for sharing and working with electronic documents - the shared data space is either small or is compressed in its visual precision.

Recent research in collaboration technology have explored shared workspaces and interaction techniques that enable data sharing within multi-display environments, such as WeSpace [7] and Impromptu [8]. These works address the co-located team meetings, including scientific collaboration situation. Inspired by these, other work, such as iBIS [9], has demonstrated a multi-display and coherent physical environment to support various interactions including distributed collaborations.

Developing technologies to support the real-world distributed scientific has been considered as necessary to understand and support the communication aspect and the dynamic of information exchange [10]. A successful collaboratory must respect users' existing communication and work mechanism. An understanding of how collaboration work should be done prior to the design and users' involvement in the design can not only allow rapid development cycle [11] but also influence the adoption of technologies and long-term outcomes which are the criteria for evaluating collaboratories [12].

Furthermore, Hollan and Stornetto [13] pointed out, that communication and collaboration tools should provide solutions "which are not ideally met in the medium of physical proximity, and evolving mechanisms which leverage the strengths of the new medium to meet those needs". In other words, rather than mimicking and supporting existing co-located collaboration practices for distributed scenarios, the new solutions should aim for enabling distributed teams to collaborate "beyond being there". Distributed scientific collaboration and communication needs exactly this kind of tools and solutions, not only to work as good as being co-located but also to increase productivity and creativity.

In this paper, we present the design process, the technical solution and the early user experience of a collaboration platform which integrates life-size video conferencing and group interactions on a large shared workspace to support distributed scientific collaborations. Our attention in this paper is directed towards supporting scientific collaboration and communication in the unique environment of the Australian Animal Health Laboratory (AAHL) which has high level of physical containment (PC) environment. Based on the foundation of prior work in collaboration technologies, we are motivated to contribute to the research field with a case of applying an integrated communication and collaboration platform in a scientific collaboration work environment. 


\section{Background}

Australian Animal Health Laboratory (AAHL) plays an important role in animal disease diagnosis, research and policy advice in Australia. Through its ongoing research programs, AAHL is able to develop the most sensitive, accurate and timely diagnostic tests, which are critical to the success of any eradication campaign in the event of a disease outbreak. AAHL also undertakes research to develop new diagnostic tests, vaccines and treatments for both exotic and endemic animal diseases.

AAHL is a "secure" scientific laboratory which has high biocontainment facility allows safely handle a range of animal species to physical containment level three (PC3) and the highest level, PC4. These levels reflect the risks involved handling biological substances. However, this facility also poses a challenge in terms of effective, rapid communication and data sharing as when leaving the containment areas staff needs to have thorough shower and equipment needs to follow strict rules of a decontamination procedure.

Real-time interactions between scientists and sharing scientific data and resources across the barrier are critical for AAHL to provide diagnostics and research services, particularly in the context of the time pressures of an emergency disease outbreak. The effort required to go through the containment barrier introduces communication difficulties between scientists working at the different physical areas. Different groups of research and diagnostics scientists need to work together and have various data resources to work with on a regular basis, such as data from high performance microscopes, shared image data bases and electronic notebooks. Email and telephone were the common communication tools. Quite often staff had to spend time going through the containment barrier to have face-to-face meetings, or meetings need to be scheduled not only with respect to availability of staff but also to the areas they are at the time. The collaborations in this environment cannot be clearly characterized as traditional "same place" or "different place" collaboration as defined by the time-space matrix [14]. Rather the collaborations across the containment barrier need be considered as in-between: collocated and remote, relating to the context of the team working at areas of different containment level but within one social organization and one physical building.

\section{Design Process}

Our goal was to develop a platform to support scientists to effectively communicate and share information across the containment barrier. The one-year design process included a field study to understand the practice, scenario-based use case analysis, iterative design, mock-up, user testing and deploying.

\subsection{Understanding the Practice}

A field study was conducted at the beginning of the project. Based on eleven semistructured interviews with different work groups, one focus group meetings with twenty staff and four site visits, we tried to build a picture of users' work practice, particularly collaborations. We had regular meetings with users in the project period and invited 
users to review and test the design before the platform was delivered. Users' continuous involvement in the design was part of the iterative design process. These understandings led to the development of three central user scenarios which helped to discuss the features of the platform between the design team and the users [15].

The diagnostics and research work in AAHL are not confined to one specific work group and often involve both staff working in the containment areas and staff working in the general office area. For example there are meetings each week between the diagnostics management team (such as the veterinary officers) who work in the general office area and scientists who work in the containment area. The veterinary officers are responsible for delivering timely technical report to state or territory animal disease control authorities. It is necessary for the veterinary officers to analyse and interpret the results together with the diagnostics scientists and microscope scientists who conduct diagnostics tests inside the PC3 area. Similarly the science research work often involves research scientists outside the PC3 area, diagnostics scientists and microscopy scientists in the PC3 areas and requires efficient data sharing between them.

During our site visits, we observed the constraints on the collaboration practice in this laboratory. The containment barrier not only separates team members spatially, but also makes their workflow difficult to organise, for example to join a meeting outside, scientists inside have to "shower out" and therefore need to schedule their activities to avoid unnecessary pauses in their work and to avoid having multiple showers a day. User scenarios have been carefully identified after discussions with the key representative staff of AAHL. The proposed scenarios address the communication and data sharing issues caused by the specific barrier, particularly the collaborative group meeting in which a group a scientists inside the containment area to work with a group scientist in the general office area. There are different types of meeting situations, ranging from conversational meetings to data-centred meetings and it is important to support both of these requirements. The technical solution (see Figure 1), a shared workspace inside the containment area and a shared workspace in the general office area, was designed and developed to mitigate the impact of moving between the two areas, to facilitate communication and to support the free flow of information and application sharing.

\subsection{Iterative Design and Testing}

At the beginning of the design process, a technical demonstration based on our previous work [9] was set up at our premise and tested by two design representatives from AAHL. Based on their hands-on experience, they were able to quickly contribute to requirement specification which helped us to generate an initial technical solution. During the design phase, different layouts, size and position of the equipment were carefully evaluated by the technical design team using existing hardware components and digital mock-up and animations. Devices were carefully chosen and tested for sufficient quality. After these steps, a refined design of the "shared workspace" was reviewed by three design representatives from AAHL and two experts from the national biosecurity authority. This feedback of the expert users were taken into account before the platform was finalised, developed and commissioned. After the introduction of the platform and training to the AAHL staff, a user study was conducted to capture the early adoption feedback from users (see section 5). 


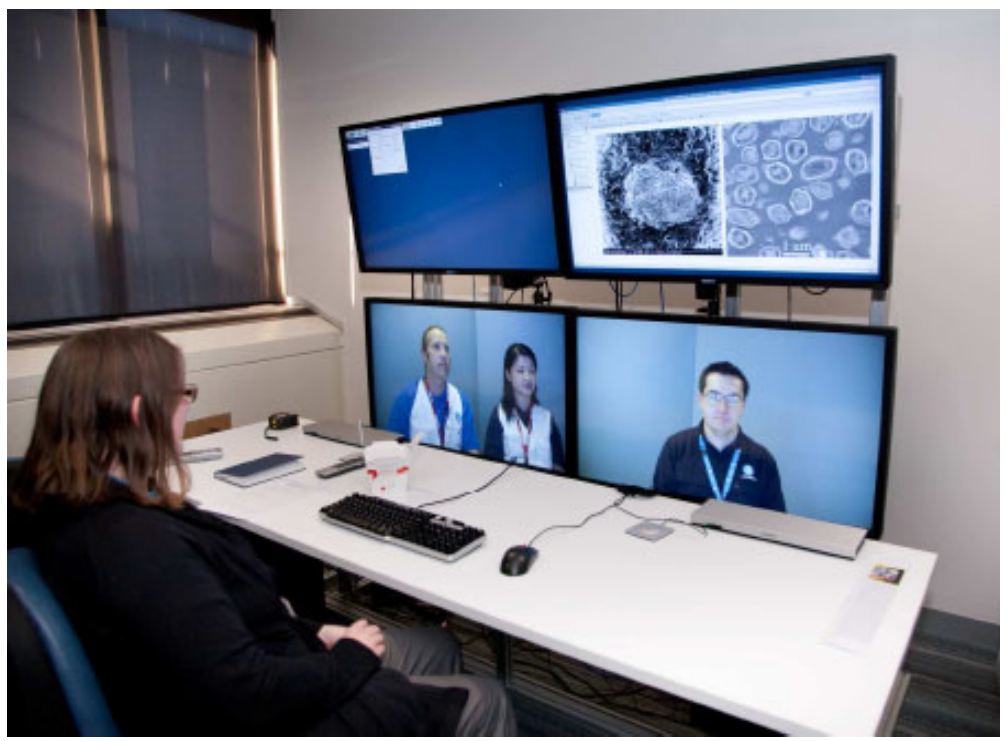

Fig. 1. The integrated communication and collaboration platform at Australian Animal Health Laboratory (AAHL)

\section{The Integrated Communication and Collaboration Platform}

In this section we describe in more details the technical aspects of the platform and explain the design rational as an outcome of the interactive process. Our considerations focused not only on the technical solutions of communication and application sharing, but also the appropriate integration of these features and the associated consideration in the design of the physical space of the platform. The communication part of the platform delivers a dual HD video link between two platforms including high quality, echo-cancelled audio communication. In addition the 8.3 Million Pixels computer workspace can be shared and displayed across the sites.

The hardware components of the platform itself are available off-the-shelf and software is mostly open source. However the close integration and flexible control of functionality of the platform are unique and to the best knowledge of the authors not achieved elsewhere, either by available products nor by related prototype from the research community. In the following sections, we will describe the physical design of the platform, and the integrated communication and collaboration technologies, as well as central user interface to the platform.

\subsection{Physical Design of the Platform}

We designed the platform in accordance with standard work place ergonomics. The table in front of the display is long enough to accommodate up to 4 users (see Figure 1). The table height of $72 \mathrm{~cm}$ and depth of $75 \mathrm{~cm}$ are those of standard office tables. The display panels with a resolution of 52 pixels per inch (ppi) suggest an optimal viewing distance of $1.66 \mathrm{~m}$ with respect to normal visual point acuity of 
users. Therefore, the complete display space lies within the limits of the users' visibility for all seating positions at the table, approx. $1.25 \mathrm{~m}$ away from the display. To avoid uncomfortable viewing angles to the top displays we decided to lower the all display as much as possible and to tilt the top displays (see Figure 2). We provided a keyboard and three mouse input devices to allow standard interaction with the computer. Two video cameras are positioned to capture all four users sitting at the table. Therefore, readjusting the cameras' orientations or field of views are not required, sitting at the table implicitly results in the fact that the users are "in the picture" (similar to systems described in [6]). In that way the overall affordance of the setting comprises the affordances of media spaces (as described in [16]) with those of the physical office environment including the chairs and the table. The table also allows to be brought into the meetings all sorts of artefacts such as pen and paper, laptops, or coffee mugs, and to place them on it.

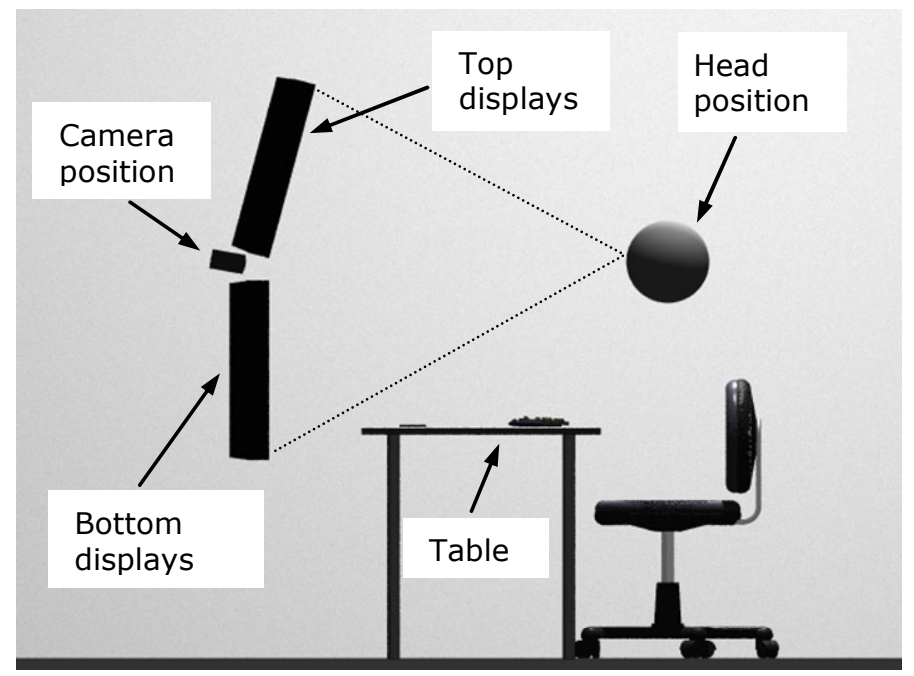

Fig. 2. The orthographic side view of the physical setting of the platform as shown in Figure 1. The bottom displays are lowered below the table surface to allow the top displays to be in a comfortable viewing position for the users.

The platform consists of four 42 inch diagonal Liquid Crystal Displays (LCD) units each with a pixel space of $1920 \times 1080$. Hence, the total display space is $3840 \times 2160$ pixels (approx. 8.3 Million Pixels) at a physical resolution of 52 ppi. All four displays are driven by one computer. The bottom two displays are mounted vertically on a frame structure and lowered to the height of $60 \mathrm{~cm}$ above the ground to allow the top displays to be lowered as well for comfortable viewing. The top displays are also mounted on the frame structure and tilted to further support a comfortable viewing by users sitting at the table. A gap between the edge of the table and the bottom displays guarantees that users sitting at the table can easily oversee the whole display space (see Figure 2). Digital models of the platform components have allowed 
us to assess design variants during the design process and helped us to develop the platform to the current state.

\subsection{Video Conferencing}

We use two off-the-shelf videoconferencing units per site to deliver the video and audio link between two sites. In a $10 \mathrm{~cm}$ gap between the two rows of displays we mounted two High Definition (HD) cameras that capture the users sitting in from of the displays. The camera positions provide good eye contact perception when the remote site is displayed on the bottom displays. The two HD cameras create a near life-size video image on the remote displays and are arranged to maintain spatial continuity of the images. Two microphones positioned on the table and connected to one conferencing unit capture the users' voices on each site.

There are three remote video display modes, "full view", "picture-in-picture", and "hide" mode (see Figure 3). In the "full" mode, the entire two bottom displays are used to show the remote site. In "picture-in-picture" mode, the video of the remote site is reduced to $25 \%$ (compared to full mode) and positioned on the top displays, left and right from the middle at the lower edge of the tops displays. Finally, in the "hide" mode no remote video image is displayed, however audio communicate remains always on. These modes allow users to switch quickly between different degrees of video media richness depending on the purpose of the meeting and particular interaction situations during the meeting.

\subsection{Application Sharing}

For the shared collaboration workspace we use a VNC server running on a dedicated server computer [17]. The client computers on each side of the collaboration platform are connected via a Gigabit network connection to this server. The clients connect to the server running a VNC viewer program [18] in full screen mode. The server is running without monitors and is configured to automatically provide the shared server facilities after restart. In that sense the client computers act like kiosks, or thin clients which have the only task to display the server computer's desktop, as typical for desktop virtualization. However, in the presented setting the size of the virtualised and shared desktop of 8.3 Million Pixels is roughly four times that of a typical personal desktop computer.

To comply with the laboratory's regulations and policies with respect to accessing computer resources we set up the application sharing in the following way. The only available applications on the shared server workspace are: a) Remote access programs to desktop computer, dedicated instrument computer, and terminal server computers, or b) a general purpose web browser. Allowing only these applications to be launched on the platform's shared workspace is compliant with the computer access policies of the laboratory. The access to intranet databases or other web-based services complies with staff's existing data access security configurations. This approach provides high flexibility of visualizing multiple desktops of remote computers and applications and the same time guarantees that no unauthorized access happens. No classified data is left on the platform's shared workspace server and client computers after the meeting. 


\subsection{User Interface Integration}

In order to provide an easy use of the platform we provided a control application to access the essential functions of the platform, such as turning on or off the video connection and the workspace sharing. One design goal of the integration was to eliminate the use of the up to 6 remote controls of the two video conferencing units and the four displays and to integrate of all platform functions at the user interface level to avoid cognitive overload (see Figure 3). Another design goal was to provide users various ways to control the platform, either through a central Graphical User Interface application with all functions or through the multi-media buttons of the computer keyboard for functions such as displays on/off or changing the remote video display mode.

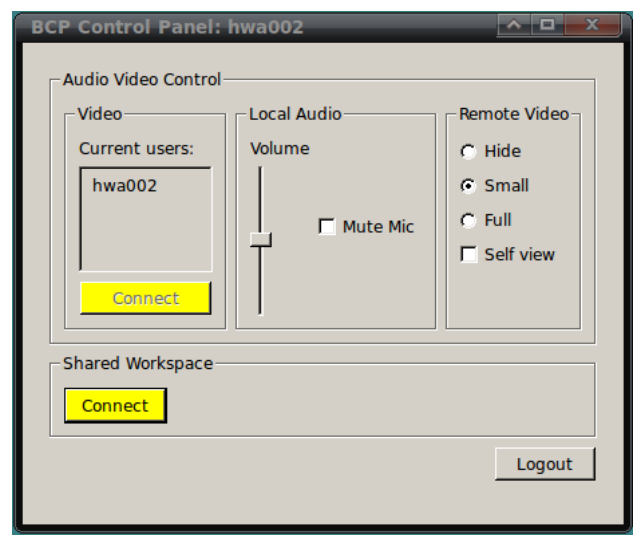

Fig. 3. The central user interface to control the platform. The interface provides all necessary functions and replaces all device remote controls.

\section{Early User Experience}

At the time of writing, the two platforms have been used at AAHL by various work groups for nearly half a year. Some of the groups have used the platform on a routine basis, other in a more ad hoc way. We conducted a user study to understand the usage of the platform in AAHL after it was used for two months. Based on a combination of interview, questionnaire and observation methods, the user study tried to capture the users' early experience with the platform.

21 staff from five work groups took part in the study. This was around $50 \%$ of the total number of staff in the five work groups who were regular or potential platform users. These participants were identified as staff who have used the platform. 5 semistructured interviews were conducted. One collaboration meeting was observed with audio-video recording of the meeting.

The results of our questionnaire and interviews have shown that study participants clearly articulated that the platform was a useful tool to enhance the communication and to enable efficient sharing information across the biocontainment barrier. 
The features of the ability of sharing and interacting with multiple data resources and high quality audio and video have been highlighted by the study participants.

The result of the questionnaires reveals that usually the number of the meeting participants were 3 to 4 at each end and the meetings lasted around 50 minutes. Most of the meetings were planned regular group meetings while some meetings were ad hoc and for special purposes. The participants rated the platform as 'easy to use' (2.4) based on a 5-point rating scale (1: very easy, 3: medium and 5; very hard). When asked about the usefulness and helpfulness of the platform compared to face-to-face meetings the average user reported "the same" (2.9) on 5-point rating scale (1: much worse, 3: the same, and 5: much better). Some participants commented that they felt that the system was better than fact-to-face because of the real-time access to and the availability to share and collaborate on all relevant data during the meetings. Although some participants felt that it can "never replace fact-to-face meetings", they valued the platform as "from a cost perspective, it helps". Our findings showed that $40.9 \%$ of the participants found that the choice of display mode depended on the situation during the meeting. This reflects the design requirement of supporting different types of working interactions (e.g. conversational meetings or data-centred meetings) and a flexible configuration of people view on the displays.

In the interviews and the questionnaires, study participants gave comprehensive feedback to the questions of what they "liked and not liked" about the platform. This feedback captured their understanding of the platform and highlighted areas for improvement. It was pointed out as a drawback that the current platform only allows one person to control the mouse at a time. Being able to work on documents in a private workspace before sharing them on the shared workspace was also mentioned as an area for improvement since there might be some sensitive documents or applications which participants may not want to share without preparations. Study participants have expressed strong interests in extending the current platform to support other collaboration scenarios, such as the collaborations with research partners outside AAHL. We also found that some users tend to use the platform for co-located meetings and make use of the large display space to share multiple personal desktops for discussions.

\section{Discussion and Future Work}

Early user experience with the platform has confirmed our design rationale of the platform. The platform meets the specific requirements of the distributed scientific collaborations in this laboratory. Users received well the benefits of the integrated platform and conceptualised the potential of a real-time information sharing in supporting their collaborative work. The feature of sharing and interacting with a broad range of data resources in large displays support the fundamental focus of scientific collaborations, particularly group collaborations. Together with the ability to quickly reconfigure the display space, the platform may support user experience that can be characterised as beyond being there. In other words, the platform not merely brings together scientists separated by the containment barrier, but also provides at the same time a flexible data sharing and communication environment that can be adjusted to different communication and collaboration needs during the scientific meetings. 
The fact that users also use the platform for co-located meetings without making use of the videoconferencing capabilities may also provide evidence for the usefulness of the platform and worth to have further investigations.

We will conduct field observations and survey over a period of several months in a longitudinal study to have in-depth understanding of the platform usage and user experience. As part of the continuous iterative design process, the areas for improvement identified from the user study will help us to refine the platform. For example, we will extend the current use scenario by providing laptop computers at each site for managing private workspaces that can be shared to the platform in an ad hoc way. Also, we hope that the integrated collaboration and communication platform we have designed and developed can be extended to support the research and operational collaborations between AAHL and a number of other organizations.

\section{Conclusion}

We described the design rationale and technical details of an integrated platform for scientific collaboration and communication. In the design process we engaged the potential user group to provide an effective technical solution to fit into the particular scientific laboratory environment. Early user feedback supports our design goal and we expect to confirm this trend further in a longitudinal study. Our design solution differs from other systems in the fact that the platform provides users with a large shared workspace for real-time collaboration in combination with high quality videoconferencing. The integrated platform bears the potential to be applicable to other domains, where distributed communication and collaboration on scientific data is the key of the application.

Acknowledgments. This work is part of a National eResearch Architecture Taskforce (NeAT) project, supported by the Australian National Data Service (ANDS) through the Education Investment Fund (EIF) Super Science Initiative, and the Australian Research Collaboration Service (ARCS) through the National Collaborative Research Infrastructure Strategy Program. We thank other members of the technical team from CSIRO ICT Centre for their contributions and Martyn Jeggo from AAHL, John Taylor from CSIRO CMIS for their support.

\section{References}

1. Wulf, W.A.: The national collaboratory: A white paper. In: Appendix A in Toward a National Collaboratory, 1 (1989); unpublished report of a National Science Foundation invitational workshop held at Rockefeller University

2. Finholt, T., Olson, G.: From laboratories to collaboratories: A new organizational form for scientific collaboration. Psychological Science (1), 28-36 (1997)

3. Olson, G.M., Zimmerman, A., Bos, N.: Scientific Collaboration on the Internet. MIT Press, Cambridge (November 2008)

4. Chen, M.: Leveraging the asymmetric sensitivity of eye contact for videoconference. In: Proceedings of the SIGCHI Conference on Human Factors in Computing Systems (CHI 2002), pp. 49-56. ACM, New York (2002) 
5. Chin Jr., G., Lansing, C.S.: Capturing and supporting contexts for scientific data sharing via the biological sciences collaboratory. In: Proceedings of the of the ACM Conference on Computer Supported Cooperative Work, CSCW 2004, pp. 409-418. ACM, New York (2004)

6. Gorzynski, M., Derocher, M., Mitchell, A.S.: The Halo B2B Studio. In: Harrison, S. (ed.) Media Space $20+$ Years of Mediated Life. Computer Supported Cooperative Work, pp. 357-368. Springer, London (2009)

7. Jiang, H., Wigdor, D., Forlines, C., Shen, C.: System design for the wespace: Linking personal devices to a table-centered multi-user, multi-surface environment. In: Tabletop, pp. 97-104. IEEE, Los Alamitos (2008)

8. Biehl, J.T., Baker, W.T., Bailey, B.P., Tan, D.S., Inkpen, K.M., Czerwinski, M.: Impromptu: a new interaction framework for supporting collaboration in multiple display environments and its field evaluation for co-located software development. In: Proceeding of the Twenty-Sixth Annual SIGCHI Conference on Human Factors in Computing Systems (CHI 2008), pp. 939-948. ACM, New York (2008)

9. Broughton, M., Paay, J., Kjeldskov, J., O’Hara, K., Li, J., Phillips, M., Rittenbruch, M.: Being here: designing for distributed hands-on collaboration in blended interaction spaces. In: Proceedings of the 21st Annual Conference of the Australian Computer-Human Interaction Special Interest Group (OZCHI 2009), pp. 73-80. ACM, New York (2009)

10. Henline, P.: Eight collaboratory summaries. Interactions, 66-72 (May 1998)

11. Finholt, T.A.: Evaluation of electronic work: Research on collaboratories at the University of Michigan. SIGOIS Bull., 49-51 (December 1995)

12. Sonnenwald, D.H.: Expectations for a scientific collaboratory: a case study. In: Proceedings of the International ACM SIGGROUP Conference on Supporting Group Work (GROUP 2003), pp. 68-74. ACM, New York (2003)

13. Hollan, J., Stornetta, S.: Beyond being there. In: Proceedings of the SIGCHI Conference on Human Factors in Computing Systems (CHI 1992), pp. 119-125. ACM, New York (1992)

14. Baecker, R.M., Grudin, J., Buxton, W., Greenberg, S.: Readings in Human-Computer Interaction: Toward the Year 2000. Morgan Kaufmann, San Francisco (January 1995)

15. Li, J., Müller-Tomfelde, C., Hyatt, A.: Supporting collaborations across a biocontainment barrier. In: Proceedings of the 2010 Conference of the Computer-Human Interaction Special Interest Group (CHISIG) of Australia on Computer-Human Interaction (OZCHI 2010), Brisbane, Australia, pp. 320-323 (November 2010)

16. Gaver, W.W.: The affordances of media spaces for collaboration. In: Proceedings of the 1992 ACM Conference on Computer-Supported Cooperative Work (CSCW 1992), pp. 1724. ACM, New York (1992)

17. TightVNC: VNC-Compatible Free Remote Control / Remote Desktop Software (2010), http: / /www.tightvnc.com/ (cited 23.01.2010)

18. RealVNC - VNC remote control software (2010), http: / / www. realvnc. com (cited 23.01.2010) 